\title{
Psoriatic Skin Lesions after Apalutamide Treatment
}

Fumi MIYAGAWA, Nobuya AKIOKA, Noriko YOSHIDA, Kohei OGAWA and Hideo ASADA

Department of Dermatology, Nara Medical University School of Medicine, 840 Shijo, Kashihara, Nara 634-8522, Japan. E-mail: fumim@ naramed-u.ac.jp

Accepted Feb 22, 2022; Epub ahead of print Feb 22, 2022

Acta Derm Venereol 2022; 102: adv00659. DOI: $10.2340 /$ actadv.v102.858

Apalutamide is a next-generation androgen receptor inhibitor, which has significantly improved the survival of prostate cancer patients $(1,2)$. However, high incidence rates of skin rashes $(23.8 \%$ and $27.1 \%)$ have been reported during apalutamide treatment $(1,2)$. We report here a case in which psoriatic skin lesions appeared after administration of apalutamide. The amelioration of the skin rash after the discontinuation of the drug and its reappearance after re-administration of the drug indicated that apalutamide was the causative agent. As low serum testosterone levels have been reported in male patients with psoriasis (3-5), this case highlights a previously unreported cutaneous adverse event of apalutamide treatment, which might be related to hormonal imbalances.

\section{CASE REPORT}

A 79-year-old Japanese man with prostate cancer was referred to us to have skin eruptions, which had persisted for 4 months, evaluated. A rash had appeared on the patient's forearm 94 days after the initiation of oral apalutamide ( $240 \mathrm{mg}$ /day) treatment and gradually spread to his entire body. Topical corticosteroids did not ameliorate the rash. He was treated with intensitymodulated radiation therapy for prostate cancer before starting apalutamide. Other medications (levetiracetam, famotidine, and magnesium oxide) were administered for 2 years. His medical history included benign brain tumour, which was surgically removed 2 years previously. The patient and his family had no history of psoriasis and/or psoriatic arthritis. On examination, he had asymptomatic, widespread, sharply demarcated, scaly erythema on his scalp, face, trunk, and extremities (Fig. 1a, b). Histological examination revealed hyperkeratosis, parakeratosis, acanthosis with elongated rete ridges, a Munro microabscess, and dilated blood vessels in the dermal papillae (Fig. 1c). These findings were compatible with psoriasis. Apalutamide was discontinued, and topical corticosteroids/vitamin D3 was initiated. The skin rash improved rapidly, but phototherapy with narrow-band UVB once a week was initiated because the patient wanted to rapidly resume apalutamide treatment upon the complete resolution of the skin rash. The rash improved further and had completely resolved within 1 month (Fig. 1d, e). One week after resolution of the rash, apalutamide treatment was restarted at a decreased dose $(120 \mathrm{mg} /$ day $)$. Five days later, the patient noticed

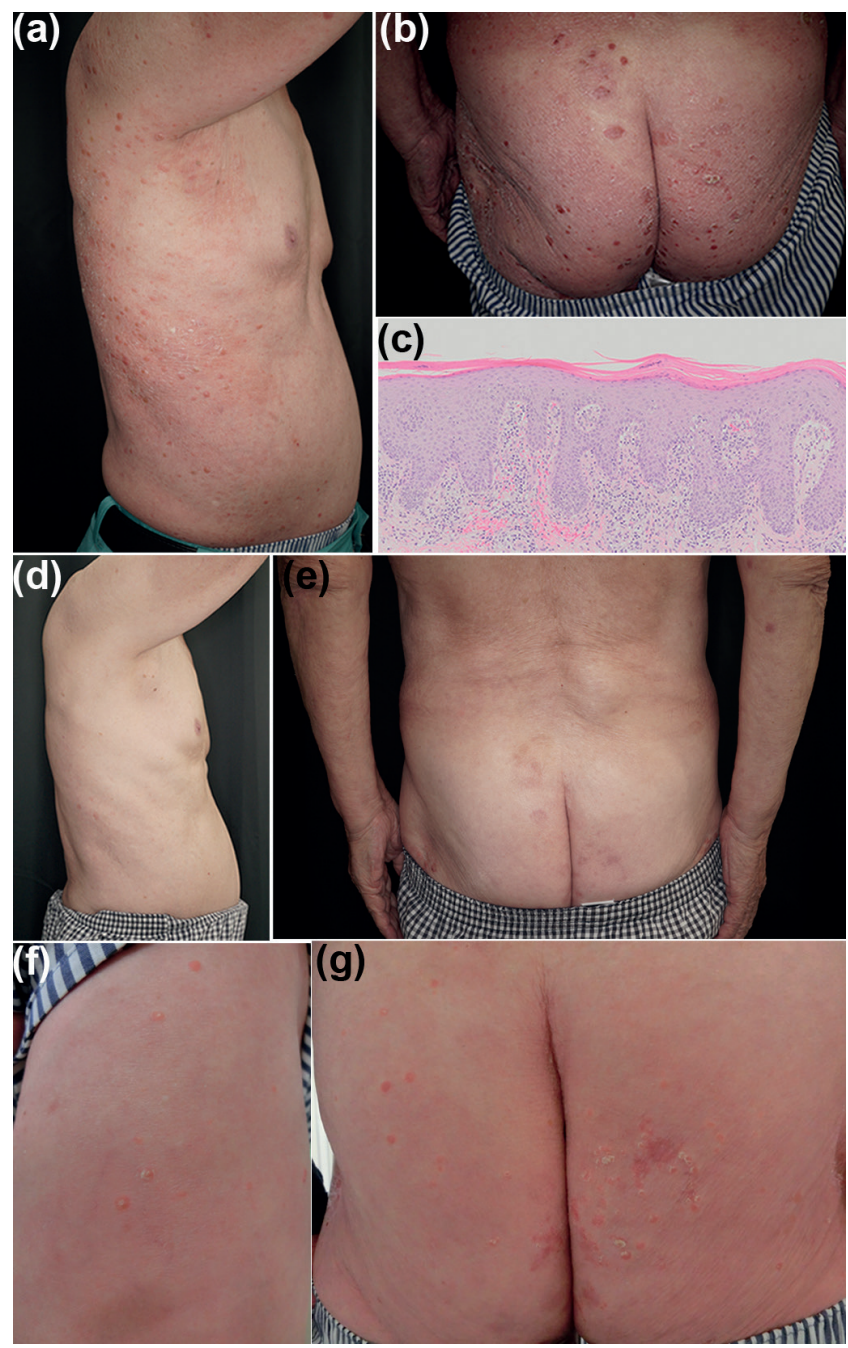

Fig. 1. (a, b) Sharply demarcated erythematous scaly papules and plaques were present on (a) the trunk and upper extremities and (b) the buttocks. (c) The skin biopsy specimen showed psoriasiform epidermal hyperplasia with hyperkeratosis and parakeratosis. In addition, there were telangiectatic blood vessels in the papillary dermis together with perivascular lymphocytic infiltrates (haematoxylin-eosin; original magnification $\times 100)$. (d, e) The rash completely resolved after discontinuation of apalutamide. Images of the trunk (d) and buttocks (e) are shown. ( $f, g)$ Erythematous scaly plaques recurred on ( $f$ ) the right thigh and $(g)$ the buttocks after the readministration of apalutamide.

a few scaly erythematous lesions on his left buttock and left thigh. Treatment with topical corticosteroids/ vitamin D3 was resumed, but the skin rash continued to spread (Fig. 1f, g). We are currently managing the skin eruptions with topical corticosteroids/vitamin D3, which have been partially effective, but new rashes continue 
to develop. A recent laboratory examination showed a decreased serum testosterone level (3.6 [normal range $131-871 \mathrm{ng} / \mathrm{dl}])$.

Written informed consent for publication of the clinical images was obtained from the patient.

\section{DISCUSSION}

Skin rashes associated with apalutamide are commonly described as macular or maculopapular $(6,7)$, but other forms of cutaneous adverse reactions, including toxic epidermal necrolysis, lichenoid drug eruptions, and urticaria, have also been reported (7). The median duration of treatment at the onset of such skin rashes is 82 days (6). To the best of our knowledge, this is the first report of psoriatic skin lesions arising after apalutamide treatment. Although the eruptions were not macular or maculopapular, our patient developed a skin rash within 3 months, and the previously reported eruptions developed within a similar timescale (6).

The pathomechanism underlying the psoriatic skin lesions that developed in the case described here is unclear. It has been reported that the serum levels of testosterone, a type of androgen, of patients with psoriasis were significantly lower than those of healthy controls (3-5), and severe psoriasis is associated with low serum testosterone levels $(4,5)$. The current patient's serum testosterone level was extremely low. Thus, one plausible explanation is that the apalutamide-induced inhibition of androgen signalling causes sex hormone imbalances, which may trigger psoriasis. Although further cases are needed to establish a causal link and understand the pathogenesis of the condition, this case may provide new insights into the molecular mechanisms of psoriatic reactions.

The authors have no conflicts of interest to declare.

\section{REFERENCES}

1. Smith MR, Saad F, Chowdhury S, Oudard S, Hadaschik BA, Graff JN, et al. Apalutamide treatment and metastasisfree survival in prostate cancer. N Engl J Med 2018; 378: 1408-1418.

2. Chi KN, Agarwal N, Bjartell A, Chung BH, Pereira de Santana Gomes AJ, Given R et al. Apalutamide for metastatic, castration-sensitive prostate cancer. N Engl J Med 2019; 381: 13-24.

3. Schwarz W, Schell H, Hornstein OP. Testosterone serum levels in male psoriatics. Arch Dermatol Res 1981; 270: 377-379.

4. Cemil BC, Cengiz FP, Atas H, Ozturk G, Canpolat F. Sex hormones in male psoriasis patients and their correlation with the Psoriasis Area and Severity Index. J Dermatol 2015; 42: $500-503$

5. Allam JP, Bunzek C, Schnell L, Heltzel M, Weckbecker L, Wilsmann-Theis $D$ et al. Low serum testosterone levels in male psoriasis patients correlate with disease severity. Eur J Dermatol 2019; 29: 375-382.

6. Rathkopf DE, Scher HI. Apalutamide for the treatment of prostate cancer. Expert Rev Anticancer Ther 2018; 18: 823-836.

7. Shima K, Nomura T, Yamada Y, Usui S, Kobayashi T, Kabashima K. Maculopapular-type drug eruptions caused by apalutamide: case series and a review of the literature. J Eur Acad Dermatol Venereol 2022; 36: e113-e115. 